\title{
Sostenibilidad curricular: construir programas académicos integrales
}

\author{
Alejandro Higuera Zimbrón, Erika Rivera Gutiérrez y Arturo Santamaría Ortega
}

\begin{abstract}
Resumen
El presente artículo tiene como objetivo exponer grosso modo un marco referencial sobre el constructo de la sostenibilidad curricular en la educación superior. Este documento se apoya en las aportaciones de algunos científicos sobre el estado actual del concepto, sin entrar a profundidad en su análisis, pues lo que se pretende es dar a conocer, a la comunidad en general, la correlación de planes de estudio y la sostenibilidad.
\end{abstract}

Palabras clave: sostenibilidad curricular, educación superior.

\section{CURRICULAR SUSTAINABILITY: BUILDING COMPREHENSIVE ACADEMIC PROGRAMS}

\begin{abstract}
The objective of this article is to present a frame of reference concerning the implementation of sustainability in higher education's curriculum. This document is based on the contributions of some scientists on the current state of the concept, however we do not deepen on it, since we intend to make known the correlation of study plans and sustainability to the general community.
\end{abstract}

Keywords: curriculum for sustainability, higher education. 
"Sostenibilidad curricular en educación superior"

\section{Alejandro Higuera Zimbrón}

ahigueraz@uaemex.mx https://orcid.org/0000-0002-7851-7531

Miembro del Sistema Nacional de Investigadores. Perfil deseable PRODEP-SEP. Profesor Investigador en la Facultad de Arquitectura y Diseño de la Universidad Autónoma del Estado de México. Adjunct Faculty Nova Southeastern University. Autor de artículos científicos en materia de Desarrollo Sostenible, Diseño y Educación. Asesor Consejos Consultivos para el Desarrollo Sustentable (SEMARnAT). Asesor para la LV y LVI legislatura en el Estado de México. Analista Internacional Senado de la República en México. Doctor en Educación por la Nova Southeastern University (USA). Maestro en Ciencias en Manejo Sostenible de Recursos por la Universidad Técnica de Múnich en Alemania. Licenciado en Relaciones Internacionales por la Universidad de las Américas Puebla.

\section{Erika Rivera Gutiérrez}

fad_graf@yahoo.com.mx

https://orcid.org/0000-0001-6966-2721

Profesora Investigadora de Tiempo Completo de la Facultad de Arquitectura y Diseño de la UAEMéx. Embajadora del Diseño Latino en México. Directora de Instituciones Incorporadas. Subdirectora Académica de la Facultad de Arquitectura y Diseño, de la UAEMéx (2010-2012).Doctora en Educación por la Nova Southeastern University en Estados Unidos de Norteamérica. Maestra en Administración de Empresas. Licenciada en Diseño Gráfico, por la Universidad Autónoma del Estado de México (UAEMéx). Miembro del Sistema Nacional de Investigadores. Perfil deseable PRODEP-SEP 2018-2022.

\section{Arturo Santamaría Ortega}

arturo_santamaria@hotmail.com asantamariao@uaemex.mx https://orcid.org/0000-0003-0866-0006

Miembro del SNI C. Doctor en Diseño. Maestría en Ingeniería -Sistemas de Manufactura- Especialidad en Diseño Estratégico de Productos Industriales. Licenciatura en Diseño Industrial. Autor de diversos artículos, libros y capítulos, conferencista magistral y ponente en diversos congresos. Profesor Investigador de Tiempo Completo de la Facultad de Arquitectura y Diseño en la UAEMéx. Coordinador del Centro de Investigación en Arquitectura y Diseño (CIAD). 
"Sostenibilidad curricular en educación superior"

\section{El rumbo hacia la sostentabilidad curricular}

El asunto de la sostenibilidad curricular es una respuesta ante la crisis ambiental causada por las actividades humanas durante los últimos años. ${ }^{1}$ Está basada en tres dimensiones: la económica, la social y la ambiental. La económica se centra en el problema de la distribución de la riqueza, ya que 20\% de la población mundial capta 86\% de la riqueza en el mundo (Azapagic, Emsley, Hamerton, 2006). Por otra parte, la social se enfoca en los beneficios del sistema relacionados con aspectos culturales y tecnológicos, a pesar de que la sociedad está menos educada y más vigilada por el gobierno junto con las corporaciones (Chomsky, 2010). La ambiental aborda cómo el planeta está manifestando reacciones extrañas a causa de la sobreexplotación de materias primas y el consumo exacerbado, aunque, paradójicamente, han disminuido las fuentes de empleo (World Watch Institute [wwl], 2014).

Autores como Larson (2012) afirman que todo surgió durante los años sesenta y setenta a partir de los reportes descritos en The silent spring de Rachel Carson y Limits of Growth de Donella Meadows, dos clásicos que anunciaron las primeras repercusiones del aumento de la población, industrialización, contaminación ambiental, producción de alimentos y agotamiento de recursos naturales en el medio ambiente. En consecuencia, se generaron alternativas desde diversas esferas, públicas, privadas y sociales, en aras de contrarrestar sus efectos.

Quizá la más importante es la de la Comisión Mundial sobre Desarrollo y Medio Ambiente (WCED). En el informe Our Common Future se expuso la primera conceptualización sobre el desarrollo sostenible, el cual se define como aquel que busca satisfacer las necesidades de la presente generación, sin afectar o comprometer las necesidades de las futuras generaciones (United Nations [UN], 1987). Dicho concepto, sin embargo, ha generado especulaciones por considerarse multifacético, sin abordar la complejidad de los sistemas.

Asimismo, en el siglo pasado se impulsaron varios foros internacionales, pero el que sentó las bases de este ejercicio fue la Cumbre de Río de Janeiro Brasil, en el año 1992. Ahí comenzó el plan de acción Agenda 21, diseñado para la implementación de estrategias que equilibren la balanza en los aspectos

1 El presente documento surge de la necesidad de exponer el marco referencial sobre la sostenibilidad curricular para los estudios superiores o de media superior. Lo anterior, desde el marco de la Educación para la Sostenibilidad (EaD), iniciativa impulsada por la Organización de las Naciones Unidas para la Educación, la Ciencia y la Cultura (UNESCO). económicos, sociales y, principalmente, ambientales a nivel mundial. El documento fue redactado en 40 capítulos, el 36 estuvo dirigido al fomento a la educación, la capacitación y la toma de conciencia. En concreto, en la fracción segunda se menciona la reorientación de la educación hacia el desarrollo sostenible (un, 1992).

Poco después, en el año 2004, se lanzó el Plan de la Década de la Educación para la Sostenibilidad. Su enfoque consistió en un marco estratégico basado en mejorar el acceso a una educación básica de calidad, aumentar el conocimiento 
del público e impartir su formación, y reorientar los programas educativos existentes hacia la sostenibilidad.

El planteamiento se confirmó en 2014, durante la Conferencia Mundial sobre la Educación para el Desarrollo Sostenible, en la declaración denominada Aichi Nagoya, en Japón. Estuvo centrada en cinco acciones prioritarias para el año 2018: promover políticas, dotar de autonomía a los jóvenes y movilizarlos, instar a las comunidades locales y las autoridades municipales a que elaboren programas de educación para la sostenibilidad de base comunitaria, aumentar las capacidades de los educadores y formadores, e integrar las prácticas de la sostenibilidad en los contextos pedagógicos y de capacitación.

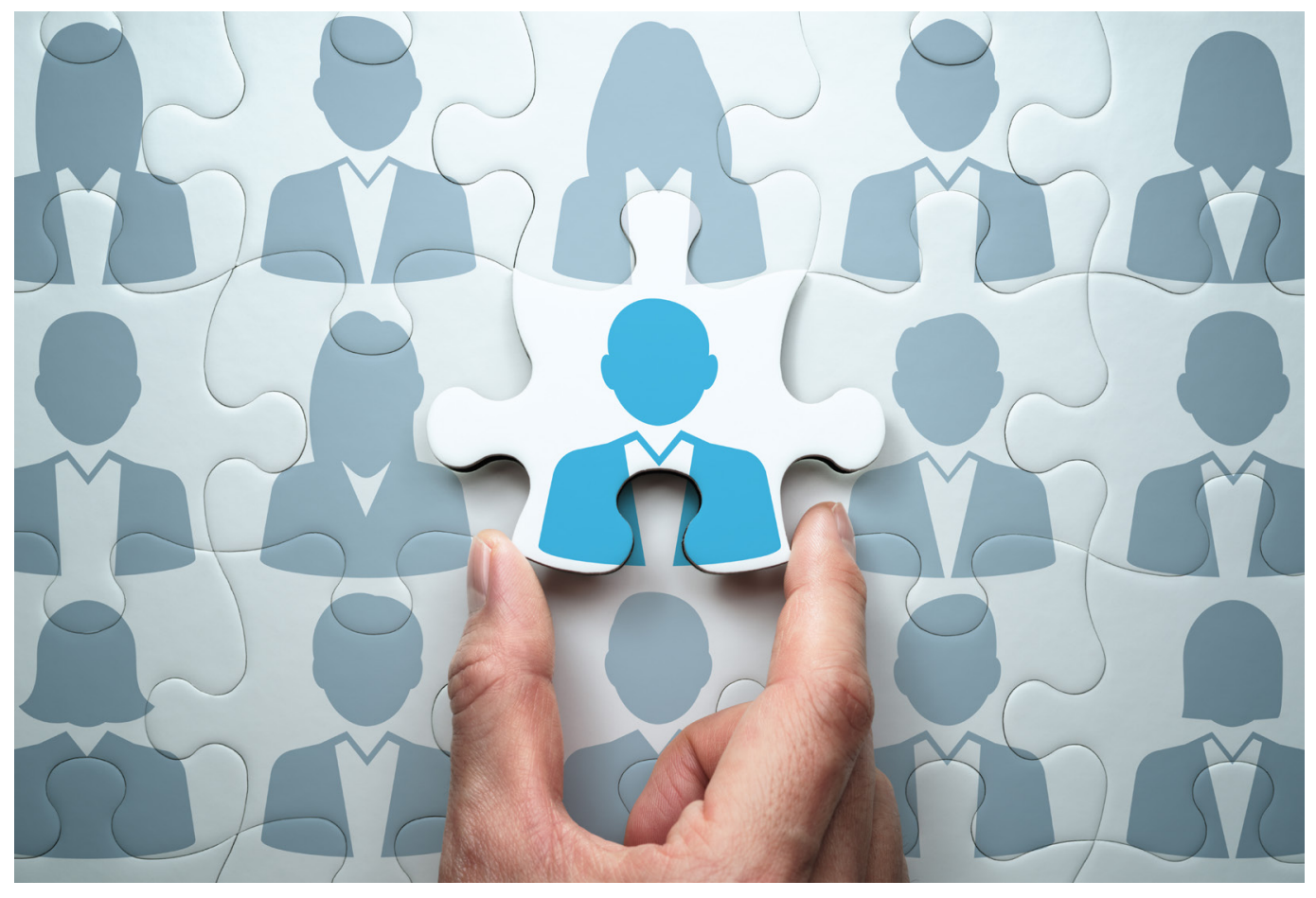

Por tanto, este documento es una aportación social basada en la necesidad de crear conciencia de la insostenibilidad planetaria entre los estudiantes universitarios. Antes de ahondar sobre los múltiples estudios que se podrían desarrollar o ya se han implementado a lo largo de dos décadas, resulta imprescindible mostrar cuáles son los planteamientos conceptuales de la sostenibilidad curricular, con el fin de comprender su significado e implicaciones.

Gunzelmann (2013) arguye, por ejemplo, que la educación para el desarrollo sostenible corresponde a una nueva visión del mundo, donde cada uno tiene la posibilidad y el compromiso, mediante la educación, de establecer nuevos modelos de vida, conductas y valores para crear un futuro mejor. Otros como Cebrian y Grace (2013) añaden que en el campo de la universidad se vislumbra la multidisciplinariedad y la interdisciplinariedad que convergen en el 
aprendizaje. Todo como un estímulo para encontrar soluciones en el presente y preparar el camino para el porvenir.

Desde otra perspectiva, Sibbel (2009) agrega que el nivel de estudios superior permanece como un reino complejo que envuelve estudiantes, académicos y administradores. Punto de encuentro en el cual se manifiestan las diversas culturas, actitudes, habilidades, experiencias, conocimientos, programas de estudio. Estos planes usualmente buscan la transformación de los alumnos a través del descubrimiento y de la generación de conocimiento gracias a la teoría, las técnicas y los métodos empleados. Por lo tanto, los programas curriculares deben obedecer a las necesidades sociales y son los estudiantes quienes en corto plazo asumirán la responsabilidad más importante en la sociedad: el progreso.

En esta misma línea, Mochizuki y Fadeeva (2010) confirman que, en general, la educación se toma como una importante herramienta de transformación para alcanzar la sostenibilidad. No obstante, ese cambio debe estar basado en una estructura bien definida entre todos los sectores sociales, particularmente el académico. Banga Chhokar (2013) advierte que la educación superior debe crecer en sus retos para poder conducir un desarrollo sostenible. Respecto a la independencia, propone que la toma de decisiones es de vital importancia. Probablemente resalte este punto, ya que la educación superior debe estar por encima de los intereses particulares y más bien enfocarse en los intereses generales.

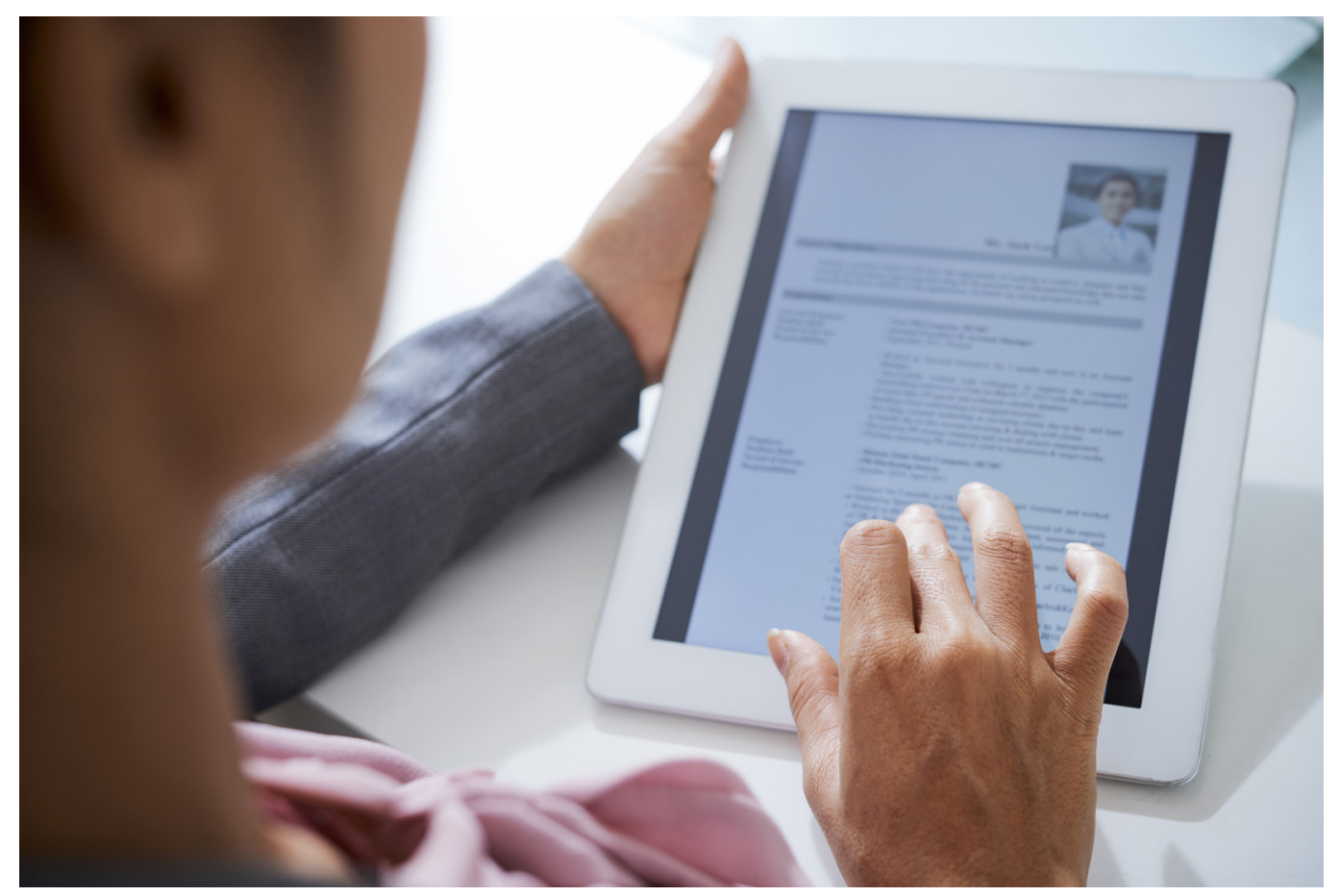


"Sostenibilidad curricular en educación superior"

Lucas (2012) dijo con razón que las escuelas requieren trabajar en las agendas de investigación e integrar los temas de sostenibilidad en los programas curriculares. Los nuevos escenarios conllevan grandes cambios de tipo económico, comercial, social, organizacional e incluso ambiental; de tal magnitud que los profesionistas tienen el reto de redefinir conceptos esenciales de las disciplinas para poder responder con efectividad y rapidez a las dinámicas del contexto. De este modo, Reunamo y Pipere (2011) sostienen que la investigación deberá reinventarse, correlacionándose con elementos holísticos, sobre todo provenientes de la necesidad social y de los cambios individuales, siempre menos materialista y más idealista.

Por su parte, Brundiers y Wiek (2010) revelan que, durante la pasada década, las instituciones de educación superior (IES) introdujeron la investigación hacia la sostenibilidad como una adhesión o anexo curricular. Sin embargo, la ciencia todavía obedece a los intereses de las grandes corporaciones y no de la sociedad. Incluso, sostienen que los patrones del neoliberalismo y consumo han causado la catástrofe natural. También se ha mencionado cómo en algunas escuelas se enseña a gastar o producir, más no a concientizar sobre la racionalidad y la conservación. No se valora en términos orgánicos, como sostiene Zygmunt Bauman en Vida líquida (2012), más bien se estima en términos de la dialéctica materialista.

Por lo anterior, Aramburuzabala, Cerrillo y Tello (2015) presentan una alternativa desde el método, El aprendizaje-servicio: una propuesta metodológica para la introducción de la sostenibilidad curricular en la Universidad. Se trata de un estudio de caso, centrado en presentar el aprendizaje-servicio como un método de enseñanza-aprendizaje. Los estudiantes y docentes no se limitan a reflexionar sobre la sostenibilidad, sino que la desarrollan en la práctica, focalizándose en algún ámbito concreto, en el que de modo efectivo y real mejora el estado de aquello para lo que cultivan y promueven, en alguna medida, el desarrollo sostenible.

Bajo esa tesitura, encontramos a los investigadores Gutiérrez-Pérez y Perales-Palacios (2012), Lozano et al. (2008), Aznar, Ull, Piñero y Martínez (2014), y González Aragón (2012), quienes proponen trabajar sobre la formación con base en las competencias de la sostenibilidad curricular. Es decir, el currículo debería contener metodologías y prácticas desde diversas perspectivas, pero siempre orientadas a los temas sociales, económicos y ambientales, en especial en dos asuntos, los sistemas de prevención y la solución de problemas. Todo, explícitamente, debería formar parte de una serie de competencias sostenibles que se destaquen en el perfil de los títulos de las diferentes carreras. 
"Sostenibilidad curricular en educación superior"

\section{Conclusiones y recomendaciones}

Tomando en cuenta este contexto, la sostenibilidad curricular en programas académicos es aún escasa y poco conocida. Se dice que muchos planes de estudios no incorporan elementos de la sostenibilidad curricular como parte fundamental del proceso de enseñanza-aprendizaje, incluso que no existe congruencia en los elementos del programa académico y aquello que la caracteriza.

Esa condición afecta a muchas instituciones, no sólo administrativamente - la institución deja de percibir apoyos económicos y orientación curricular por parte de la unESCO-, también académicamente por dos razones. Primera, porque genera un rezago en el establecimiento de políticas relacionadas con la sostenibilidad curricular: no ha habido una contribuición al plan de la educación para la sostenibilidad formulada por la unEsco desde los años 2004, 2014 y hasta el 2018. Segunda, debido a que los estudiantes no están participando en la solución de problemas in situ, lo que los convirte en alumnos inoperativos, pues no son agentes de cambio y viven aislados de la realidad.

Además, se afirma que la universidad debe fomentar la participación de su comunidad, junto con otros elementos como sociedad, industria y gobierno, en la solución de los problemas tanto locales como globales (Higuera, 2018). En otras palabras, mientras la universidad no adapte sus planes curriculares a la realidad, difícilmente se tendrá una comunidad de egresados consciente de los retos futuros.

En tanto la sostenibilidad curricular contempla una serie de características que deberán estar plasmadas en todos los programas académicos con una base de "realidad", sólo se detectan aproximaciones. En el caso de los programas de educación superior, se percibe que se trabaja bajo un esquema determinado por un modelo que ya se encuentra muy dañado en lo académico.

Finalmente, se recomienda que los estudiantes, quienes son los portadores de las nuevas ideas, reciban un conocimiento basado en hechos reales, cuantificables y calificables (herramientas cualitativas). Se necesita que el conocimiento esté justificado en los problemas actuales de insostenibilidad planetaria, además de estimular a la innovación y al desarrollo, así como a la solución de problemas. Quizá existen múltiples interrogantes sin respuestas sencillas, tal vez lo real es que no hay una contribución académica en relación con la educación para la sostenibilidad, aún un asunto sin agotar, propenso para nuevas investigaciones. 
"Sostenibilidad curricular en educación superior" Alejandro Higuera Zimbrón, Erika Rivera Gutiérrez y Arturo Santamaría Ortega Vol. 21, Núm. 3, mayo-junio 2020 Revista Digital Universitaria

\section{Referencias}

* Aramburuzabala, P, Cerrillo, R, Tello, I. (2015). Aprendizaje-servicio: una propuesta metodológica para la introducción de la sostenibilidad curricular en la Universidad. Profesorado, Revista de Curriculum y formación del profesorado, 19(1), 81. http:// www.ugr.es/ recfpro/rev191ART5.pdf.

* Azapagic, A., Emsley, A. y Hamerton, L. (2003). Polymers, the environment and sustainable development. John Wiley \& Sons, Ltd.

* Aznar Minguet, P. y UII, M. A. (2009). La formación de competencias básicas para el desarrollo sostenible: el papel de la Universidad. Revista de Educación. Número extraordinario 2009, 219-237. http://www.revistaeducacion.mec.es/inicio.html.

* Brundiers, K., y Wiek, A. (2011). Educating students in real-world sustainability research: Vision and implementation. Innovative Higher Education, 36(2), 107-124. https://doi.org/10.1007/s10755-010-9161-9.

* Cebrián, G., Grace, M., y Humphris, D. (2013). Organisational learning towards sustainability in higher education. Sustainability Accounting, Management and Policy Journal, 4 (3), 285-306. http://dx.doi.org/10.1108/SAMPJ-12-2012-0043.

* Chhokar, K. B. (2010). Higher education and curriculum innovation for sustainable development in India. International Journal of Sustainability in Higher Education, 11(2), 141-152. http://dx.doi.org/10.1108/14676371011031865.

* Chomsky, N. (2010). Esperanzas y realidades. Urano.

- González Aragón, Carmen (2012). Sostenibilidad Curricular en los Planes de Estudios de la Universidad de Cádiz [tesis de maestría, Universidad de Cádiz. España]. http:// rodin.uca.es/xmlui/bitstream/handle/10498/16564/Sostenibilidad Curricular en los Planes de Estudios de la Universidad de Cádiz.pdf? sequence=1.

* Gutiérrez-Pérez, J., Perales-Palacios, F. J. (2012). Ambientalización curricular y Sostenibilidad. Nuevos retos de Profesionalización docente. Profesorado, Revista de Curriculum y formación del profesorado, p. 6. http://www.ugr.es/local/recfpro/ rev162ed.pdf.

- Gunzelmann, B. (2013). Global voices and global visions: Education for excellence, understanding, peace and sustainability. Rowman and Littlefield Publishers.

* Higuera, Z. A. (2018). Método de la Tetra Hélice (Industria, Gobierno, Sociedad y Educación) como factores de evaluación en los Procesos de Acreditación en México. Actas de Diseño, xill Encuentro Latinoamericano de Diseño, ix Congreso Latinoamericano de Enseñanza del Diseño, 25. Consultado el 17 de abril de 2019. https://fido.palermo.edu/servicios dyc/encuentro2010/administracionconcursos/archivos conf 2013/882 57494 981con.pdf.

* Larson, B. A. (2012). Campus Leadership's Influence in Implementing a Community College's Sustainability Goals: A Case Study [tesis doctoral, Nova Southeastern University]. https://pqdtopen.proquest.com/doc/1170811361.html?FMT=Al.

* Lucas, F. J. (2012). Higher Education for sustainability: cases, challenges, opportunities. From across the curriculum. Routledge. 
"Sostenibilidad curricular en educación superior" Alejandro Higuera Zimbrón, Erika Rivera Gutiérrez y Arturo Santamaría Ortega Vol. 21, Núm. 3, mayo-junio 2020 Revista Digital Universitaria

* Lozano-García, F. J., Gándara, G., Perrni, O., Manzano, M., Elia Hernández, D. y Huisingh, D. (2008). Capacity building: A course on sustainable development to educate the educators. International Journal of Sustainability in Higher Education, 9(3), 257-281. http://dx.doi.org/10.1108/14676370810885880.

* Mochizuki, Y. y Fadeeva, Z. (2010), Competences for sustainable development and sustainability, International Journal of Sustainability in Higher Education, 1, 391-403. http://dx.doi.org/10.1108/14676371011077603.

* Reunamo, J. y Pipere A. (2011). Doing research on education for sustainable development. International Journal of Sustainability in Higher Education, 12, 110-124. http://dx.doi.org/10.1108/14676371111118183.

- Sibbel, A (2009). Pathways towards sustainability through higher education. International Journal of Sustainability in Higher Education, 10, 68-82. http://dx.doi. org/10.1108/14676370910925262.

* Ull, M. A., Martínez Agut, M. P., Piñero, A. y Aznar Minguet, P. (2010). Análisis de la introducción de la sostenibilidad en la enseñanza superior en europa: compromisos institucionales y propuestas curriculares. Revista Eureka sobre Enseñanza y Divulgación de las Ciencias, 7, número extraordinario, La sosenibilidad en la universidad, 413-432. http://ojs.uca.es/index.php/tavira/article/viewFile/56/54.

* United Nations (Un). (1992). Programa de Agenda 21. http://www.un.org/spanish/ esa/sustdev/agenda21/index.htm.

* United Nations for Education, Culture \& Science Organization (unEsco). (2004). Decenio de la educación para la sostenibilidad. http://www.unesco.org/new/ es/unesco-world-conference-on-esd-2014/esd-after-2014/global-actionprogramme/launching/.

* United Nations Education, Culture \& Science Organization (Unesco). (2014). Declaración deAichi Nagoya. http://www.unesco.org/new/es/media-services/singleview/news/world conference on education for sustainable development calls for renewed commitment by all countries/ - VMKBn0eG91Y.

* Vilches A, Gil Pérez. (2013). Ciencia de la sostenibilidad: Un nuevo campo de conocimientos al que la química y la educación química están contribuyendo. Educación química, 24(2), 199-206. http://www.scielo.org.mx/pdf/eq/v24n2/v24n2a4.pdf.

* World Watch Institute (wwI). (2014). State of the World: Is Sustainability Still Possible? http://www.worldwatch.org/bookstore/publication/state-world-2013sustainability-still-possible.

\section{Cómo citar este artículo}

- Higuera Zimbrón, Alejandro, Rivera Gutiérrez, Erika y Santamaría Ortega, Arturo. (2020). Sostenibilidad curricular: construir programas académicos integrales. Revista Digital Universitaria (RDU) Vol. 21, núm. 3 mayo-junio. Dol: http://doi.org/10.22201/ codeic.16076079e.2020.v21n3.a10

Recepción: 24/05/2019. Aceptación: 06/02/2020. 